\section{Disparidade racial na sobrevivência em 10 anos para o câncer de mama: uma análise de mediação usando abordagem de respostas potenciais}

\author{
Racial disparity in 10-year breast cancer survival: \\ a mediation analysis using potential responses \\ approach
}

\section{Disparidad racial en la supervivencia en 10 años para el cáncer de mama: un análisis de mediación usando un enfoque de respuestas potenciales}

Mário Círio Nogueira 1

Maximiliano Ribeiro Guerra 1

Jane Rocha Duarte Cintra 2

Camila Soares Lima Corrêa 1

Vivian Assis Fayer 1

Maria Teresa Bustamante-Teixeira 1

doi: 10.1590/0102-311X00211717

\title{
Resumo
}

Os objetivos foram investigar a associação entre raça/cor e a sobrevivência em 10 anos de mulheres com câncer de mama e o papel do estadiamento como mediador. Coorte hospitalar com 481 mulheres com câncer invasivo de mama, diagnosticadas entre 2003 e 2005. Foram feitas comparações entre mulheres brancas e negras quanto às características sociodemográficas e ao estadiamento, usando o teste qui-quadrado, e à sobrevivência em 10 anos, usando os métodos de Kaplan-Meier e regressão de Cox. Foram estimados para a variável raça/cor efeitos diretos e indiretos, mediados pelo estadiamento, com ajuste para a condição social da área de residência e idade, utilizando o modelo de respostas potenciais (contrafactual) e regressão múltipla de Cox. As mulheres negras residiam em setores censitários de menor renda, eram usuárias do setor público em maior proporção e foram diagnosticadas com estadiamentos mais avançados. A sobrevivência específica em 10 anos foi de 64,3\% (IC95\%: 60,0; 68,9), com diferença significativa entre brancas (69,5\%; IC95\%: 64, 8; 74,6) e negras (44\%; IC95\%: 35,2; 55,1). Nos modelos múltiplos, ajustados para renda e idade, as negras tiveram pior prognóstico (HR = 2,09; IC95\%: 1,76; 2,51), e a proporção mediada pelo estadiamento foi de 40\% (IC95\%: 37; 42). Há disparidade racial na sobrevivência do câncer de mama em 10 anos, mediada principalmente pelo estadiamento mais avançado da doença nas mulheres negras. Isso aponta para a necessidade de ampliar a cobertura e a qualidade do programa de rastreamento dessa doença e facilitar o acesso ao diagnóstico e tratamento precoces, com vistas à redução da iniquidade racial.

Equidade em Saúde; Origem Étnica e Saúde; Neoplasias da Mama; Análise de Sobrevida

\author{
Correspondência \\ M. C. Nogueira \\ Departamento de Saúde Coletiva, Faculdade de Medicina, \\ Universidade Federal de Juiz de Fora. \\ Rua Alberto Vieira Lima 357, Juiz de Fora, MG 36.050-070, \\ Brasil. \\ mario.cirio.nogueira@gmail.com \\ 1 Faculdade de Medicina, Universidade Federal de Juiz de Fora, \\ Juiz de Fora, Brasil. \\ 2 Instituto Oncológico, Juiz de Fora, Brasil.
}




\section{Introdução}

A neoplasia de mama é responsável pelo maior número de óbitos femininos por câncer em países em desenvolvimento, como o Brasil 1. Nos Estados Unidos, as disparidades raciais no prognóstico de mulheres com essa doença são bem estabelecidas 2,3,4, enquanto, no Brasil, os poucos trabalhos publicados sobre o tema apresentaram resultados divergentes 5,6,7,8,9.

Os principais determinantes sociais que aparecem nos estudos de sobrevivência por câncer de mama são a posição social, mensurada de diferentes maneiras, e a raça/cor. Em relação a essa última, existem dificuldades conceituais e metodológicas, relacionadas à definição e à mensuração da variável raça/cor, e também aos métodos de análise e inferência causal 10,11. Apesar disso, a presença de grandes iniquidades raciais em algumas populações justifica essa linha de investigação 12 .

As pesquisas epidemiológicas tomam a raça não como um conceito biológico, mas como uma construção social, um produto da história e da cultura humanas, com origens no colonialismo e na escravidão. A definição de raça pela cor da pele, como é feita no Brasil, em comparação a critérios de ancestralidade ou etnia, é mais influenciável pelo contexto social, econômico e cultural 13.

$\mathrm{Na}$ análise estatística de modelos múltiplos em epidemiologia, quando estão sendo investigados determinantes do processo saúde-doença, é importante utilizar um quadro conceitual que aponte as relações hierárquicas entre os determinantes. Se isso não for feito, pode-se subestimar o efeito dos determinantes mais distais 14 . Se uma variável de exposição é intermediária na relação causal entre uma variável mais distal e o desfecho, ela não se caracteriza como variável de confusão, e sim como mediadora do efeito da exposição sobre o desfecho, e o ajuste por ela só será adequado nas análises de mediação causal. Confundimento, que é um conceito causal, não pode ser detectado ou corrigido apenas por métodos estatísticos, mas deve estar baseado nas premissas causais 15 .

São poucos os estudos de mediação causal que utilizam a abordagem contrafactual na investigação de disparidades raciais e sociais no prognóstico de pacientes com câncer de mama 3,16, não tendo sido identificado nenhum com dados brasileiros. Essa abordagem é a mais adequada aos estudos de decomposição do efeito da exposição em seus componentes direto e indireto. Uma das razões para a carência de estudos nessa área é a dificuldade metodológica, pois, apenas recentemente, estratégias analíticas mais válidas foram desenvolvidas 17.

O objetivo deste estudo foi investigar a associação entre raça/cor e a sobrevivência em 10 anos em uma coorte de mulheres com câncer de mama, analisando o papel do estadiamento como possível mediador dessa associação, por meio do modelo contrafactual.

\section{Métodos}

\section{População de estudo}

Coorte de base hospitalar composta por 563 mulheres com câncer de mama, diagnosticadas entre 2003 e 2005, e acompanhadas em Unidade de Assistência de Alta Complexidade em Oncologia (UNACON), em um Município de Minas Gerais, Brasil, que atende pacientes dos setores público e privado e é referência para uma macrorregião do estado.

\section{Instrumentos e procedimentos}

A identificação das pacientes foi feita inicialmente a partir do registro de câncer de base hospitalar da instituição. As informações foram extraídas dos prontuários por especialistas em oncologia, utilizando ficha padronizada e, quando necessário, complementadas por contato telefônico.

Das 563 mulheres iniciais da coorte, 46 foram excluídas por não se tratar de câncer invasivo; das 517 restantes, outras 36 foram excluídas por não serem residentes no Estado de Minas Gerais, sendo 35 residentes no Rio de Janeiro e uma residente na Bahia. Essa exclusão foi necessária porque a informação sobre o desfecho óbito foi obtida do banco de mortalidade de Minas Gerais (Sistema de Informações sobre Mortalidade - SIM/MG). A população efetivamente analisada correspondeu a 481 mulheres. 
A data inicial do estudo foi 1o de janeiro de 2003, e o início do tempo de seguimento de cada paciente correspondeu à data do diagnóstico histopatológico do câncer de mama. Para o seguimento de 10 anos, os seguintes procedimentos foram adotados, nesta ordem: consulta ao SIM/MG; consulta aos prontuários hospitalares; ligação telefônica; contato com os mastologistas de referência das pacientes; consulta ao Cadastro Nacional de Falecidos - CNF (https://www.falecidosnobrasil.org. br/; acessado em Mar/2017); consulta à situação cadastral do Cadastro de Pessoas Físicas (CPF https://www.situacaocadastral.com.br/; acessado em Mar/2017), com identificação do ano do óbito, quando presente. A maioria das pacientes teve seu seguimento completado pelos quatro primeiros procedimentos. Apenas nove pacientes tiveram sua data de óbito definida pelo CNF, e 39 pacientes tiveram sua situação de vivas ao fim do seguimento definida pela situação cadastral do CPF. Para validar a informação proveniente do CPF, foi realizada análise comparativa entre essa informação e a do estado vital confirmada por outras fontes para as 201 pacientes com ambas as informações. Dessa forma, o valor preditivo estimado do cadastro do CPF para identificação da paciente como viva foi de 95\%, enquanto, para confirmação do óbito, foi de 100\%. Após todas essas etapas, $17(3,5 \%)$ pacientes permaneceram com seguimento de 10 anos incompleto.

Este estudo foi autorizado pelo Comitê de Ética em Pesquisa da Universidade Federal de Juiz de Fora, município sede do hospital - parecer no 1.006.551.

\section{Modelo conceitual e variáveis coletadas}

Foi utilizado modelo conceitual hierarquizado, que considerou como determinantes sociais distais raça/cor e renda domiciliar per capita média do setor censitário de residência da mulher. A idade da paciente no momento do diagnóstico também foi considerada como determinante distal, por poder influenciar a renda e o acesso ao rastreamento. A idade foi categorizada em três faixas, tendo como pontos de corte 50 e 69 anos, por ser o intervalo preconizado para rastreamento mamográfico no Brasil. Como determinantes sociais intermediários, foram selecionados natureza do setor onde foi tratada (pública ou privada) e atraso no início do tratamento superior a 60 dias após o diagnóstico. Como fator proximal e variável de mediação dos fatores mais distais na determinação da sobrevivência, foi estudado o estadiamento do tumor (classificação TNM: I, II, III e IV).

A raça foi categorizada em branca ou negra, essa última resultante da agregação das categorias preta e parda. A informação foi obtida por meio de contato telefônico com a própria paciente ou com um familiar, no caso de já ter ocorrido o óbito. A opção pela agregação das categorias preta e parda decorreu da semelhança entre as mulheres dessas categorias em relação às demais variáveis e ao prognóstico nesta amostra, da frequência relativa menor dessas categorias em relação à categoria branca e do fato de as políticas públicas de enfrentamento das desigualdades raciais no Brasil tratarem esses segmentos de maneira conjunta ${ }^{18}$. A renda domiciliar per capita média do setor censitário de residência da mulher foi obtida do Censo Demográfico 2010 do Instituto Brasileiro de Geografia e Estatística (IBGE) e categorizada em quartis, identificados como renda baixa, média, alta e muito alta.

\section{Análise dos dados}

\section{- Definições}

A data do diagnóstico histopatológico da doença foi considerada o início do tempo de sobrevivência, e o desfecho, como o óbito em consequência do câncer de mama ou de seu tratamento, estimando a sobrevivência específica por câncer de mama. Para os 19 casos de óbitos com causa não definida, considerou-se como causa o câncer de mama, com o intuito de se obter uma estimativa mais conservadora da sobrevivência específica 19.

Os desfechos ocorridos dentro dos 10 anos iniciais após o diagnóstico foram considerados como falhas. Foram censurados as mulheres que permaneceram vivas até essa data, os óbitos ocorridos por outras causas e os casos considerados como perda de seguimento. Esses últimos foram censurados na data referente ao último acompanhamento identificado. Para cada paciente, os tempos máximos de seguimento considerados foram de 10 anos. 


\section{- Análises exploratórias}

Inicialmente, foram descritas as distribuições das variáveis pelas categorias de raça/cor, usando o teste qui-quadrado (ou o exato de Fisher quando indicado) para testar associações, considerando-se estatisticamente significativas se valor de $\mathrm{p}<0,05$. Como outro método exploratório, foram calculadas as probabilidades de sobrevivência em 10 anos pelo método de Kaplan-Meier, com intervalo de $95 \%$ de confiança (IC95\%), estratificadas por cada variável de exposição. Foi construído um gráfico das curvas de sobrevivência específica por câncer de mama estratificada por raça/cor. Em seguida, também usando as curvas de Kaplan-Meier, foram estimados os tempos de sobrevivência médios restritos (restricted mean survival time - RMST) com IC95\% 20.

\section{- Modelos múltiplos}

Para avaliar a associação entre as variáveis de exposição e a sobrevivência específica por câncer de mama, foi usada a abordagem hierarquizada convencional ${ }^{14}$, com modelos de regressão de Cox múltiplos, iniciando com o modelo apenas com raça/cor, ajustado pela idade, com acréscimo sucessivo de variáveis, do nível mais distal ao nível mais proximal, mantendo o ajuste pela idade. Para essa etapa da análise, foram excluídas as mulheres com dados faltantes para as variáveis raça/cor ( 9 pacientes) e renda média do setor censitário (13 pacientes). O pressuposto de proporcionalidade dos riscos foi avaliado pela análise dos resíduos de Schoenfeld. O ajuste dos modelos foi avaliado pelo R2 ajustado, pela log-verossimilhança e pela análise gráfica dos resíduos deviance e escore 21.

Para a análise de mediação causal, foi utilizada a abordagem contrafactual, usando a classe de modelos NEM (natural effect models) 17. Tem como pressupostos o controle dos fatores de confusão para a relação entre exposição e desfecho, entre exposição e variável mediadora, e entre mediadora e desfecho. O controle das variáveis de confusão foi feito de acordo com o modelo conceitual: ajuste para as variáveis idade e renda média do setor censitário de residência, ambas do mesmo nível hierárquico da exposição. Considerando a permutabilidade condicional entre expostos e não expostos, dada pelo controle das variáveis de confusão, as medidas de associação podem ser interpretadas como medidas de efeito. Outro pressuposto dos modelos NEM é a ausência de interação entre exposição e mediadora. Um terceiro pressuposto é a positividade, que indica a necessidade de probabilidade maior que zero para cada nível de exposição, estratificado para cada nível dos fatores de confusão, o que foi verificado nos dados. O último pressuposto é a consistência da exposição, também chamada SUTVA (stable unit treatment value assumption), que exige uma única versão da exposição para todos os indivíduos no estudo, ou que, pelo menos, as várias versões, se existirem, resultem no mesmo desfecho para o mesmo nível de exposição 15 .

Para definir os parâmetros a serem estimados, duas variáveis contrafactuais foram definidas 22:

$\mathrm{Y}_{\mathrm{i}}(\mathrm{a}, \mathrm{m})=$ desfecho alcançado para a pessoa i se, possivelmente contrário ao fato, a exposição foi especificada como "a" e o mediador como " $m$ ";

$\mathrm{M}_{\mathrm{i}}(\mathrm{a})=$ mediador alcançado para a pessoa " $\mathrm{i}$ " se, possivelmente contrário ao fato, a exposição foi especificada como "a".

As variáveis contrafactuais aninhadas foram definidas como: $\mathrm{Y}\left(\mathrm{a}, \mathrm{M}\left(\mathrm{a}^{*}\right)\right)$.

Considerando variáveis de exposição e mediadora dicotômicas (no caso do estadiamento, dicotomizando em I/II e III/IV) e utilizando modelos de regressão de Cox, temos as seguintes quantidades estimadas, na forma de hazard ratios (HR):

$$
\begin{aligned}
H R_{\text {total }} & =\frac{\text { hazard para } Y(1, M(1))}{\text { hazard para } Y(0, M(0))} \\
H R_{\text {direto }} & =\frac{\text { hazard para } Y(1, M(0))}{\text { hazard para } Y(0, M(0))} \\
H R_{\text {indireto }} & =\frac{\text { hazard para } Y(1, M(1))}{\text { hazard para } Y(1, M(0))}
\end{aligned}
$$

O efeito total da exposição também pode ser estimado como o produto do HR direto pelo $\mathrm{HR}$ indireto. $\mathrm{O}$ efeito natural direto é o efeito estimado ao mudar o nível da exposição, mas mantendo 
a variável de mediação fixa no nível que ela teria se não tivéssemos mudado a exposição. $\mathrm{O}$ efeito natural indireto, por sua vez, é o efeito estimado ao mudar o nível da variável de mediação como se tivéssemos mudado a exposição, mas sem mudar a exposição.

Para a implementação dos modelos NEM, foi seguido procedimento sugerido recentemente na literatura epidemiológica, com a programação de uma função no programa R (The R Foundation for Statistical Computing, Viena, Áustria; http://www.r-project.org) para a decomposição de efeitos 23. Na primeira etapa, uma regressão foi ajustada para obter as estimativas de efeito da exposição na variável mediadora, controlando para os fatores de confusão. Na segunda etapa, o banco de dados foi duplicado com diferentes valores da exposição (contrafactuais). Na etapa 3 , foram determinados pesos (Wc) para os dados replicados de acordo com a expressão a seguir:

$$
W^{c}=\frac{P\left(M \mid A^{*}, C\right)}{P(M \mid A, C)}
$$

A probabilidade $\mathrm{P}$ foi derivada de uma regressão do mediador $\mathrm{M}$ na exposição $\mathrm{A}$ e nas variáveis de confusão C. Na etapa 4, os pesos foram usados em um modelo de Cox ponderado para estimar os hazard ratios dos efeitos naturais direto e indireto e do efeito total. Os intervalos de confiança foram obtidos por reamostragem por bootstrap.

Para as análises de sensibilidade, foram realizados: testes de interação entre a variável de exposição raça/cor e a variável mediadora estadiamento; estimação de modelos com imputação dos dados faltantes para as variáveis raça/cor e renda, especificando valores extremos; estimação de modelos de sobrevivência global, especificando como desfechos os óbitos por todas as causas.

A entrada dos dados foi feita nos programas Epi Info 2002 (Centers for Disease Control and Prevention, Atlanta, Estados Unidos), e as análises, no programa R, versão 3.4.0 (https://www.r-project. org/). O código de todas as análises feitas no programa R está disponível no repositório de um dos autores no endereço eletrônico: https://github.com/marionog/Estudo-sobrevivencia.

\section{Resultados}

A Tabela 1 apresenta a distribuição das variáveis segundo as categorias de raça/cor. A faixa etária predominante foi de 50-69 anos, sem associação com raça/cor. As mulheres foram categorizadas por quartis de renda domiciliar per capita média do setor censitário de residência, e houve uma associação entre raça/cor negra e maior proporção de residência em setores censitários de menor renda. A proporção de mulheres no setor privado foi maior para a raça/cor branca e no setor público para a negra, com associação significativa. A proporção de mulheres com atraso no início do tratamento foi pequena e sem associação com raça/cor. As mulheres de raça/cor negra foram diagnosticadas com estadiamentos mais avançados no momento do diagnóstico. No período de 10 anos, a proporção de óbitos foi maior nas mulheres negras do que nas brancas, tanto por câncer de mama $(54,5 \%$ vs. 28,6\%; $\mathrm{p}<0,001)$ quanto por todas as causas $(61,4 \% v s .42 \% ; \mathrm{p}=0,001)$, embora esta análise ainda não tenha levado, em conta, a censura.

A sobrevivência específica por câncer de mama foi de 64,3\% (IC95\%: 60,0; 68,9). Tiveram menores valores de sobrevivência e de RMST as mulheres com faixas etárias menores de 50 anos e maiores que 70 anos, com raça/cor negra, com rendas mais baixas, do setor público do hospital e com estadiamentos mais avançados (Tabela 2). A Figura 1 mostra as curvas de sobrevivência específica estratificada por raça/cor.

A Tabela 3 apresenta os modelos de Cox múltiplos para a sobrevivência específica por câncer de mama, ajustados por idade. Tiveram associação independente com menor sobrevivência em 10 anos raça/cor negra, renda baixa ou muito baixa e estadiamento mais avançado. A associação entre raça/ cor negra e menor sobrevivência foi discretamente atenuada pela renda e, em maior proporção, pelo estadiamento, mantendo-se, contudo, significativa.

$\mathrm{Na}$ análise dos resíduos de Schoenfeld, os testes de correlação linear global e de cada variável dos modelos não foram significativos, indicando proporcionalidade dos riscos para todos os modelos múltiplos. Os gráficos desses resíduos pelo tempo também não mostraram violação do pressuposto de proporcionalidade. Foram observados alguns valores outliers na análise de resíduos dos modelos múltiplos, mas que não foram considerados influentes nas estimativas. 
Distribuição das variáveis por raça/cor da pele para as mulheres da coorte hospitalar de câncer de mama residentes em Minas Gerais, Brasil, diagnosticadas entre 2003 e 2005 ( $N=481)$.

\begin{tabular}{|c|c|c|c|c|c|}
\hline \multirow[t]{3}{*}{ Variáveis * } & \multicolumn{4}{|c|}{ Raça/Cor da pele } & \multirow[t]{3}{*}{ Valor de $p$} \\
\hline & \multicolumn{2}{|c|}{$\begin{array}{l}\text { Branca } \\
(\mathrm{n}=371)\end{array}$} & \multicolumn{2}{|c|}{$\begin{array}{c}\text { Negra } \\
(n=101)\end{array}$} & \\
\hline & $\mathrm{n}$ & $\%$ & $\mathbf{n}$ & $\%$ & \\
\hline Faixa etária (anos) & & & & & 0,301 \\
\hline$<50$ & 117 & 31,5 & 40 & 39,6 & \\
\hline $50-69$ & 170 & 45,8 & 42 & 41,6 & \\
\hline$\geq 70$ & 84 & 22,6 & 19 & 18,8 & \\
\hline Renda domiciliar per capita média ** & & & & & $0,005 * * *$ \\
\hline Alta & 98 & 27,1 & 13 & 13,4 & \\
\hline Média & 96 & 26,5 & 20 & 20,6 & \\
\hline Baixa & 85 & 23,5 & 31 & 32,0 & \\
\hline Muito baixa & 83 & 22,9 & 33 & 34,0 & \\
\hline Natureza do setor & & & & & $<0,001 * *$ \\
\hline Privado & 190 & 51,2 & 17 & 16,8 & \\
\hline Público & 181 & 48,8 & 84 & 83,2 & \\
\hline Atraso no tratamento $>60$ dias & & & & & 0,603 \\
\hline Não & 354 & 95,4 & 95 & 94,1 & \\
\hline Sim & 17 & 4,6 & 6 & 5,9 & \\
\hline Estadiamento do tumor & & & & & $0,001 * *$ \\
\hline 1 & 103 & 27,8 & 13 & 12,9 & \\
\hline ॥ & 147 & 39,6 & 35 & 34,7 & \\
\hline III & 90 & 24,3 & 41 & 40,6 & \\
\hline IV & 31 & 8,4 & 12 & 11,9 & \\
\hline Óbitos por neoplasias da mama & & & & & $<0,001 * *$ \\
\hline Não & 265 & 71,4 & 46 & 45,5 & \\
\hline $\operatorname{Sim}$ & 106 & 28,6 & 55 & 54,5 & \\
\hline Óbitos por todas as causas & & & & & $0,001 * *$ \\
\hline Não & 215 & 58,0 & 39 & 38,6 & \\
\hline Sim & 156 & 42,0 & 62 & 61,4 & \\
\hline
\end{tabular}

* Dados faltantes para as seguintes variáveis: raça/cor da pele (9 pacientes); renda domiciliar per capita (13 pacientes);

** Categorias de renda definidas por quartis da renda média do setor censitário de residência no Censo Demográfico 2010;

$\star \star \star$ Teste qui-quadrado (ou exato de Fisher, quando indicado); significativo se $p<0,05$.

A Tabela 4 apresenta os resultados da análise de mediação causal e da análise de sensibilidade. A raça/cor negra teve pior prognóstico na sobrevivência em 10 anos, com efeitos direto e indireto significativos. O estadiamento ao diagnóstico respondeu por 40\% (IC95\%: 37; 42) do efeito de raça/ cor (Modelo 1). Nas análises de sensibilidade, a imputação de valores extremos para os dados faltantes das variáveis raça/cor (Modelos 2 e 3) e renda (Modelos 4 e 5) não modificaram substancialmente os resultados dos modelos de mediação causal. Na análise de sobrevivência global (Modelo 6), especificando como desfecho os óbitos por todas as causas, os efeitos de raça/cor foram de menor magnitude, mas a proporção mediada pelo estadiamento foi muito próxima, estimada em 39\% (IC95\%: 29; 67). Foi ajustado modelo de regressão de Cox, com inclusão das variáveis de exposição, de mediação, de interação entre as duas e das covariáveis, e não foi detectada interação significativa entre raça/cor e estadiamento, com valor estimado do coeficiente da interação de -0,390 ( $p=0,244)$. 


\section{Tabela 2}

Sobrevivências específicas em 10 anos estimadas pelo método de Kaplan-Meier e hazard ratios (HR) estimados por regressão de Cox, estratificados por categorias das variáveis de exposição, Minas Gerais, Brasil, 2003-2005.

\begin{tabular}{|c|c|c|c|c|c|c|c|c|}
\hline \multirow[t]{2}{*}{ Variáveis * } & \multirow[t]{2}{*}{$\mathbf{n}$} & \multirow[b]{2}{*}{ Eventos } & \multicolumn{4}{|c|}{ Kaplan-Meier } & \multicolumn{2}{|c|}{ Regressão de Cox } \\
\hline & & & $\begin{array}{l}\text { Sobrevivência } \\
10 \text { anos (\%) }\end{array}$ & IC95\% & RMST & IC95\% & HR & IC95\% \\
\hline Geral & 481 & 162 & 64,3 & 60,$0 ; 68,9$ & 2.828 & $2.714 ; 2942$ & - & - \\
\hline \multicolumn{9}{|c|}{ Faixa etária (anos) } \\
\hline$<50$ & 159 & 60 & 61,6 & 54,$4 ; 69,8$ & 2.730 & $2.528 ; 2.932$ & Ref. & - \\
\hline $50-69$ & 218 & 65 & 68,2 & 62,$1 ; 75,0$ & 2.974 & $2.817 ; 3.131$ & 0,76 & 0,$53 ; 1,08$ \\
\hline$\geq 70$ & 104 & 37 & 60,8 & 51,$5 ; 71,7$ & 2.673 & $2.410 ; 2.936$ & 1,06 & 0,$70 ; 1,60$ \\
\hline \multicolumn{9}{|l|}{ Raça/Cor } \\
\hline Branca & 371 & 106 & 69,5 & 64,$8 ; 74,6$ & 2.962 & $2.840 ; 3.084$ & Ref. & - \\
\hline Negra & 101 & 55 & 44,0 & 35,$2 ; 55,1$ & 2.298 & $2.026 ; 2.570$ & 2,27 & 1,$64 ; 3,15$ \\
\hline \multicolumn{9}{|c|}{ Renda domiciliar per capita média } \\
\hline Alta & 117 & 27 & 75,4 & 67,$7 ; 84,0$ & 3.137 & $2.947 ; 3.327$ & Ref. & - \\
\hline Média & 117 & 33 & 69,9 & 61,$8 ; 79,2$ & 2.982 & $2.766 ; 3.198$ & 1,28 & 0,$77 ; 2,13$ \\
\hline Baixa & 117 & 42 & 62,5 & 54,$1 ; 72,2$ & 2.786 & $2.551 ; 3.021$ & 1,70 & 1,$05 ; 2,76$ \\
\hline Muito baixa & 117 & 55 & 49,9 & 41,$3 ; 60,3$ & 2.389 & $2.134 ; 2.644$ & 2,59 & 1,$63 ; 4,11$ \\
\hline \multicolumn{9}{|c|}{ Natureza do setor } \\
\hline Privada & 214 & 57 & 72,0 & 66,$0 ; 78,5$ & 3.031 & $2.880 ; 3.182$ & Ref. & - \\
\hline Pública & 267 & 105 & 58,2 & 52,$3 ; 64,7$ & 2.665 & $2.502 ; 2.828$ & 1,67 & 1,$21 ; 2,31$ \\
\hline \multicolumn{9}{|c|}{ Atraso no tratamento $>60$ dias } \\
\hline Não & 458 & 152 & 64,8 & 60,$4 ; 69,5$ & 2.841 & $2.725 ; 2.957$ & Ref. & - \\
\hline $\operatorname{Sim}$ & 23 & 10 & 54,9 & 37,$6 ; 80,2$ & 2.587 & $2.075 ; 3.099$ & 1,34 & 0,$71 ; 2,54$ \\
\hline \multicolumn{9}{|c|}{ Estadiamento do tumor } \\
\hline 1 & 118 & 14 & 87,1 & 81,$0 ; 93,7$ & 3.413 & $3.288 ; 3.588$ & Ref. & - \\
\hline$\|$ & 188 & 41 & 76,3 & 70,$1 ; 83,0$ & 3.191 & $3.048 ; 3.334$ & 1,95 & 1,$07 ; 3,59$ \\
\hline III & 132 & 67 & 46,6 & 38,$5 ; 56,3$ & 2.414 & $2.181 ; 2.647$ & 5,74 & 3,$23 ; 10,22$ \\
\hline IV & 43 & 40 & 7,0 & 2,$3 ; 20,8$ & 968 & $690 ; 1.246$ & 22,77 & 12,$27 ; 42,26$ \\
\hline
\end{tabular}

Eventos: óbitos por câncer de mama; IC95\%: intervalos de 95\% de confiança; Ref.: referência; RMST: tempo de sobrevivência médio restrito (restricted mean survival time), limite superior 3.653 dias.

* Dados faltantes para as seguintes variáveis: raça/cor da pele (9); renda domiciliar per capita (13).

\section{Discussão}

Este estudo investigou a disparidade racial na sobrevivência específica por câncer invasivo de mama em 10 anos e o papel do estadiamento ao diagnóstico como mediador. O prognóstico foi pior para mulheres de raça/cor negra e residentes em setores censitários com menor renda média, com efeito independente de ambas as variáveis. O efeito de raça/cor sobre a sobrevivência foi mediado parcialmente pelo estadiamento; as mulheres negras tiveram maior proporção de estádios mais avançados, característica associada a pior prognóstico.

A sobrevivência específica por câncer de mama em 10 anos nesta coorte hospitalar de mulheres diagnosticada entre 2003 e 2005 foi de 64,3\% (IC95\%: 60,0; 68,9), situando-se numa posição intermediária na comparação com outros municípios brasileiros ou com outros países. Foram identificados seis estudos de sobrevivência em 10 anos de mulheres com câncer de mama residentes no Brasil. Em estudo de base populacional em Goiânia, Goiás, com mulheres diagnosticadas entre 1988 e 1990, a sobrevivência global foi de 41,5\% 5. Outro estudo de base populacional em Goiânia, com diagnóstico da doença entre 1995 e 2003, encontrou aumento da sobrevivência global em 10 anos para 57,8\% 24 . Um terceiro estudo de base populacional feito com mulheres diagnosticadas entre 1993 e 1995 na 
Gráfico com as curvas de sobrevivência específica em 10 anos por câncer de mama estratificada por raça/cor, estimadas pelo método de Kaplan-Meier.

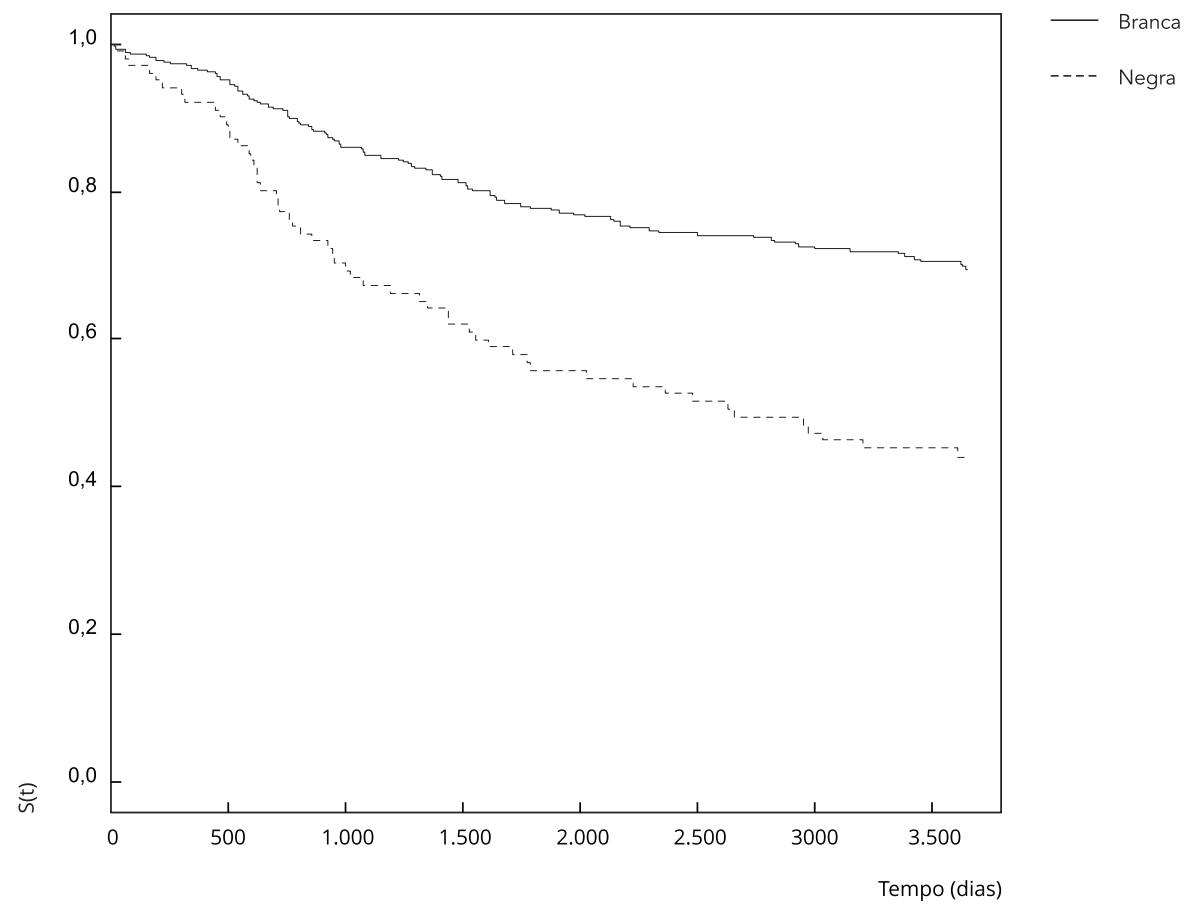

cidade de Campinas, São Paulo, estimou uma sobrevivência específica de 68,9\% em 10 anos 25. Os outros três estudos foram feitos com coortes hospitalares: em Joinville, Santa Catarina, com mulheres diagnosticadas em 2008, a sobrevivência específica foi de 83,1\% 8; em Juiz de Fora, Minas Gerais, com mulheres diagnosticadas em 2000 e 2001, a sobrevivência global foi de 56,3\% 6; em Santa Maria, Rio Grande do Sul, com mulheres diagnosticadas entre 1980 e 2000, a sobrevivência global foi de 78,7\% 26.

No Reino Unido, a sobrevivência relativa em 10 anos foi de 57,5\% para mulheres com diagnóstico no período de 1986 a 1990 e subiu para 67\% para as diagnosticadas entre 1991 e 1995 27. Entre as participantes do estudo ONCOPOOL (Pooling of European Data to Harmonise Translational Research in Breast Cancer) 28, que incluía mulheres com câncer de mama operável e diagnosticado na década de 1990 de 10 serviços oncológicos em países da Europa, a sobrevivência geral em 10 anos foi de 80\%, e a específica foi de $84 \%$. Em uma coorte hospitalar na Coreia do Sul, com pacientes diagnosticadas entre 1991 e 2005, a sobrevivência global em 10 anos foi de 80,5\% 29. Na Europa, dados populacionais de mulheres com câncer de mama diagnosticado entre 2000 e 2002, analisados no estudo EUROCARE-4 (Survival of Cancer Patients in Europe), mostraram sobrevivência relativa em 10 anos de mais de $70 \%$ na maioria dos países, com grandes diferenças internas, desde apenas $54 \%$ na região oriental até $75 \%$ na região norte da Europa 30. Percebe-se, portanto, que a sobrevivência em 10 anos das mulheres com câncer de mama vem aumentando no Brasil, assim como nos países de renda mais alta, embora ainda apresente valores menores do que nesses países. As prováveis razões do aumento da sobrevivência estão relacionadas ao aumento do rastreamento mamográfico, levando ao diagnóstico em estadiamentos mais precoces e a melhorias no cuidado das pacientes com câncer 28,30 .

As desigualdades raciais em saúde têm sido pouco estudadas no Brasil. Em parte, isso se deve à indisponibilidade de dados relativos à raça nos principais bancos de dados epidemiológicos até 


\section{Tabela 3}

Medidas de associação ajustadas - hazard ratios (HR) dos modelos de Cox múltiplos para a sobrevivência específica em 10 anos da coorte hospitalar de câncer de mama de mulheres residentes em Minas Gerais, Brasil, diagnosticadas entre 2003 e 2005 (N = 459 ).

\begin{tabular}{|c|c|c|c|c|c|}
\hline Variáveis & Modelo 1 & Modelo 2 & Modelo 3 & Modelo 4 & Modelo 5 \\
\hline \multicolumn{6}{|l|}{ Raça/Cor } \\
\hline Branca & Ref. & Ref. & Ref. & Ref. & Ref. \\
\hline \multirow[t]{2}{*}{ Negra } & 2,21 & 2,08 & 1,98 & 2,08 & 1,68 \\
\hline & $(1,58 ; 3,10)$ & $(1,48 ; 2,91)$ & $(1,39 ; 2,82)$ & $(1,48 ; 2,92)$ & $(1,19 ; 2,37)$ \\
\hline Renda domiciliar per capita média & & & Ref. & & Ref. \\
\hline Alta & & Ref. & Ref. & Ref. & Ref. \\
\hline \multirow[t]{2}{*}{ Média } & & 1,22 & 1,21 & 1,22 & 0,95 \\
\hline & & $(0,73 ; 2,04)$ & $(0,72 ; 2,02)$ & $(0,73 ; 2,05)$ & $(0,56 ; 1,60)$ \\
\hline \multirow[t]{2}{*}{ Baixa } & & 1,53 & 1,48 & 1,54 & 1,85 \\
\hline & & $(0,94 ; 2,51)$ & $(0,90 ; 2,43)$ & $(0,94 ; 2,52)$ & $(1,12 ; 3,04)$ \\
\hline \multirow[t]{2}{*}{ Muito baixa } & & 2,39 & 2,25 & 2,43 & 2,27 \\
\hline & & $(1,49 ; 3,83)$ & $(1,38 ; 3,68)$ & $(1,51 ; 3,91)$ & $(1,41 ; 3,66)$ \\
\hline \multicolumn{6}{|l|}{ Natureza do setor } \\
\hline Privada & & & Ref. & & \\
\hline \multirow[t]{2}{*}{ Pública } & & & 1,17 & & \\
\hline & & & $(0,81 ; 1,69)$ & & \\
\hline \multicolumn{6}{|l|}{ Atraso no tratamento $>60$ dias } \\
\hline Não & & & & Ref. & \\
\hline \multirow[t]{2}{*}{ Sim } & & & & 0,87 & \\
\hline & & & & $(0,43 ; 1,73)$ & \\
\hline \multicolumn{6}{|l|}{ Estadiamento } \\
\hline । & & & & & Ref. \\
\hline \multirow[t]{2}{*}{$\|$} & & & & & 1,90 \\
\hline & & & & & $(1,03 ; 3,50)$ \\
\hline \multirow[t]{2}{*}{ III } & & & & & 5,17 \\
\hline & & & & & $(2,87 ; 9,30)$ \\
\hline \multirow[t]{2}{*}{ IV } & & & & & 24,52 \\
\hline & & & & & $(12,92 ; 46,53)$ \\
\hline $\mathrm{R}^{2}$ corrigido & $4,3 \%$ & $7,6 \%$ & $7,8 \%$ & $7,6 \%$ & $31,3 \%$ \\
\hline$-2(L 0-L 1)$ & $-906,8$ & $-898,7$ & $-898,3$ & $-898,6$ & $-832,4$ \\
\hline
\end{tabular}

Nota: todos os modelos ajustados por idade.

recentemente. Outra razão importante é que as dificuldades na definição de raça e na operacionalização de sua classificação são obstáculos à pesquisa sobre o tema. Embora raça não seja uma categoria biológica válida para o ser humano, é uma construção social com impactos no acesso a recursos e na valorização na sociedade, juntamente com outros indicadores de posição social, como gênero, educação e renda. Assim, diferenças socioeconômicas acumuladas por gerações podem estar na raiz das desigualdades raciais em saúde. Além do impacto da raça na posição socioeconômica do indivíduo, outros mecanismos podem estar operando como determinantes de saúde 31 .

É de se esperar uma correlação entre a definição da raça/cor do indivíduo e o seu contexto socioeconômico. A percepção atual da raça/cor do indivíduo tem como determinante um processo histórico complexo, por sua vez com vários componentes, como o fenótipo físico do indivíduo e de seus pais, a base genética, o contexto cultural e a posição socioeconômica familiar e da vizinhança, indicando a necessidade de uso de uma ou mais variáveis representativas desses conceitos para controle de confundimento. Ao se estimar o efeito da variável raça/cor após ajuste por variável socioeconômica, uma interpretação possível é que estamos estimando a disparidade racial em saúde que persiste mesmo após a remoção das desigualdades socioeconômicas ${ }^{32}$. Esses problemas na definição e mensuração 


\section{Tabela 4}

Decomposição do efeito total de raça/cor em efeitos naturais direto e indireto (hazard ratios - HR), mediado pela variável estadiamento, e análise de sensibilidade para a sobrevivência específica em 10 anos, Minas Gerais, Brasil, 2003-2005.

\begin{tabular}{|c|c|c|c|c|c|c|}
\hline Efeitos (HR raça/Cor $)$ & Modelo 1 & Modelo 2 & Modelo 3 & Modelo 4 & Modelo 5 & Modelo 6 \\
\hline Total & $\begin{array}{c}2,09 \\
(1,76 ; 2,51)\end{array}$ & $\begin{array}{c}2,09 \\
(1,76 ; 2,51)\end{array}$ & $\begin{array}{c}2,09 \\
(1,76 ; 2,51)\end{array}$ & $\begin{array}{c}2,18 \\
(1,92 ; 2,52)\end{array}$ & $\begin{array}{c}2,18 \\
(1,92 ; 2,52)\end{array}$ & $\begin{array}{c}1,74 \\
(1,33 ; 2,16)\end{array}$ \\
\hline Direto & $\begin{array}{c}1,56 \\
(1,39 ; 1,78)\end{array}$ & $\begin{array}{c}1,56 \\
(1,39 ; 1,78)\end{array}$ & $\begin{array}{c}1,56 \\
(1,39 ; 1,78)\end{array}$ & $\begin{array}{c}1,62 \\
(1,52 ; 1,78)\end{array}$ & $\begin{array}{c}1,62 \\
(1,52 ; 1,78)\end{array}$ & $\begin{array}{c}1,40 \\
(1,10 ; 1,72)\end{array}$ \\
\hline Indireto (mediado por estádio) & $\begin{array}{c}1,34 \\
(1,27 ; 1,41)\end{array}$ & $\begin{array}{c}1,34 \\
(1,27 ; 1,41)\end{array}$ & $\begin{array}{c}1,34 \\
(1,27 ; 1,41)\end{array}$ & $\begin{array}{c}1,34 \\
(1,26 ; 1,41)\end{array}$ & $\begin{array}{c}1,34 \\
(1,26 ; 1,41)\end{array}$ & $\begin{array}{c}1,24 \\
(1,21 ; 1,25)\end{array}$ \\
\hline Proporção mediada & $\begin{array}{c}40 \% \\
(37 \% ; 42 \%)\end{array}$ & $\begin{array}{c}40 \% \\
(37 \% ; 42 \%)\end{array}$ & $\begin{array}{c}40 \% \\
(37 \% ; 42 \%)\end{array}$ & $\begin{array}{c}38 \% \\
(36 \% ; 38 \%)\end{array}$ & $\begin{array}{c}38 \% \\
(36 \% ; 38 \%)\end{array}$ & $\begin{array}{c}39 \% \\
(29 \% ; 67 \%)\end{array}$ \\
\hline
\end{tabular}

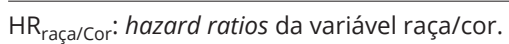

Nota: todos os modelos ajustados por idade e renda média do setor censitário de residência; os intervalos de $95 \%$ de confiança foram obtidos por bootstrap, com 10.000 replicações.

Modelo 1: feito com as 459 pacientes com dados completos.

Análise de sensibilidade:

Modelo 2: feito com 468 pacientes, imputando raça/cor branca para os casos sem essa informação (9).

Modelo 3: feito com 468 pacientes, imputando raça/cor negra para os casos sem essa informação (9).

Modelo 4: feito com 472 pacientes, imputando renda alta para os casos sem essa informação (13).

Modelo 5: feito com 472 pacientes, imputando renda muito baixa para os casos sem essa informação (13).

Modelo 6: usando óbitos por todas as causas (sobrevivência global) em vez de óbitos por câncer de mama (sobrevivência específica) como desfecho.

da raça/cor trazem uma dificuldade adicional na aplicação da abordagem contrafactual, pois um de seus pressupostos é a consistência da exposição e, no caso de raça/cor e outras características individuais, dificilmente é possível traduzi-las diretamente em intervenções hipotéticas. Por outro lado, esses estudos podem ser vistos como um passo inicial para que outros a seguir investiguem possíveis intervenções 33 .

Poucos estudos foram identificados com o objetivo de avaliar os fatores mediadores das diferenças raciais na sobrevivência de mulheres com câncer de mama usando uma abordagem contrafactual. Em estudo de coorte prospectiva nos Estados Unidos, foi encontrada menor sobrevivência para as mulheres negras comparadas com as brancas, mesmo após ajuste para características sociodemográficas, estadiamento e positividade para receptores hormonais, sendo esses dois últimos fatores responsáveis pela maior proporção de mediação; o conjunto de mediadores estudados explicou 53,9\% do excesso de risco de óbito das mulheres negras 16. Em um grande estudo feito com registro de base hospitalar nacional nos Estados Unidos, a disparidade racial na sobrevivência foi mediada por tipo de seguro de saúde, estadiamento, comorbidades e tratamento, que, combinados, responderam por $76,3 \%$ do excesso de óbitos em mulheres negras 3. Outro estudo recente sobre disparidade racial em saúde nos Estados Unidos usou a abordagem clássica, estimando o efeito mediado pela redução relativa do HR após ajustar pela variável mediadora, e encontrou uma proporção do efeito de raça/cor mediada pelo estadiamento de 23,6\% 2. Essas investigações sobre disparidades raciais em saúde feitas nos Estados Unidos podem não se aplicar inteiramente ao Brasil, por diferenças nas composições e relações étnico-raciais 12 .

Vários estudos recentes investigaram disparidades raciais na sobrevivência de mulheres com câncer de mama nos Estados Unidos, todos apresentando como resultado pior prognóstico para mulheres negras, mediado principalmente pelo estadiamento mais avançado no momento do diagnóstico 2,3,4. Nesses estudos, o pior prognóstico das mulheres negras permaneceu significativo mesmo após ajuste para idade, posição socioeconômica e estadiamento. No Brasil, por outro lado, os poucos estudos feitos sobre o tema tiveram resultados divergentes, a maioria não encontrando associação entre raça/ cor e sobrevivência 5,6,7,8 e apenas um mostrando pior prognóstico para as mulheres negras 9 . Nesse 
último, a associação permaneceu significativa mesmo após ajuste para idade e escolaridade, mas não após ajuste para estadiamento.

De forma semelhante ao nosso estudo, investigações feitas no Brasil 7,9,34 e em outros países 2,4,35,36,37,38 encontraram associação entre pior posição socioeconômica e menor sobrevivência no câncer de mama. Vários desses estudos também utilizaram medidas relativas à área de residência como indicador socioeconômico, geralmente pela indisponibilidade ou incompletude de informações individualizadas.

Embora as mulheres negras tenham tido maior proporção de atraso superior a 60 dias para o início do tratamento, isso não teve efeito na sobrevivência, provavelmente pelo baixo percentual de mulheres que sofreram tal atraso (4,8\%). Estudo feito nos Estados Unidos com mulheres entre 15 e 39 anos verificou menor sobrevivência por câncer de mama naquelas que tiveram atraso para o início do tratamento superior a seis semanas, com pior prognóstico para as afro-americanas, sem seguro-saúde e com pior condição socioeconômica 39 . Em outro estudo feito com dados dos registros regionais de câncer nos Estados Unidos, apenas com mulheres acima de 65 anos, as afro-americanas apresentaram pior prognóstico do câncer de mama, mediado, em parte, por atraso no início da quimioterapia superior a três meses após a cirurgia 40.

As mulheres de raça/cor negra foram diagnosticadas em estadiamentos mais avançados, e essa característica foi importante para lhes conferir menor sobrevivência em comparação com as brancas. Isso se deve provavelmente a menor acesso e/ou menor adesão ao rastreamento do câncer de mama por meio da mamografia, embora também possa ser, em parte, atribuído a um comportamento mais agressivo do tumor em mulheres negras. Estudo feito nos Estados Unidos com 63.472 mulheres com câncer de mama identificadas em registro regional de câncer encontrou que as afro-americanas foram diagnosticadas com idades mais precoces e doença mais avançada e tiveram menor sobrevivência. Após ajuste por estadiamento ao diagnóstico, faixa etária, posição socioeconômica, comorbidades e tratamento, as diferenças diminuíram, mas continuaram significativas. Com base nesses achados, os autores sugeriram que o rastreamento fosse iniciado em idade mais precoce para as afro-americanas 41 .

Embora em nosso estudo não tenhamos dados sobre rastreamento para câncer de mama, existem estudos de base populacional no Brasil que mostram desigualdades de acesso à mamografia de rastreamento, com maior utilização dela por mulheres brancas, de maior escolaridade e renda, com plano de saúde e residentes em municípios maiores 42 .

O estadiamento no momento do diagnóstico não explicou toda a disparidade racial na sobrevivência das mulheres com câncer de mama neste estudo. Outros fatores podem ter contribuído, pelo menos, em parte, para as diferenças verificadas na sobrevivência, como características relativas ao tumor 43 e ao tratamento 44 .

As disparidades raciais em saúde costumam ser atribuídas a diferenças socioeconômicas, mas, em muitos estudos, elas persistem mesmo após o ajuste para variáveis socioeconômicas. Por exemplo, as diferenças no estado de saúde autopercebido entre brancos e negros no Brasil, desfavoráveis aos negros, persistem mesmo quando se leva, em conta, a situação socioeconômica dos indivíduos, pelo nível de escolaridade ou de renda 45 . Uma possível explicação seria que o nível socioeconômico atual do indivíduo pode não refletir inteiramente as diferenças na exposição a fatores de risco ao longo da vida, devido ao seu contexto social. Outra questão é que variáveis individuais não refletem inteiramente o nível socioeconômico das pessoas, pois esse depende também do ambiente social onde estão inseridos, especialmente o seu contexto de trabalho e de residência 46.

Outra explicação para as desigualdades raciais em saúde verificadas nos estudos epidemiológicos refere-se à possibilidade de erros de mensuração da variável raça/cor, variáveis ao longo do tempo, do espaço ou de acordo com o método de mensuração. No entanto, algumas análises feitas com dados de vários estudos populacionais brasileiros concluíram que esses erros, embora presentes, não chegam a influenciar significativamente as estimativas de desigualdades raciais 18 .

Uma das limitações deste estudo é ser baseado em uma coorte hospitalar. No entanto, trata-se de uma unidade hospitalar de referência regional, com assistência pública e privada e, dessa forma, potencialmente representativa do conjunto das mulheres com câncer de mama. Outra limitação relaciona-se ao uso de dados secundários de diversas fontes, como o prontuário hospitalar e o SIM, com a possibilidade de erros de informação, minimizados pelos procedimentos adotados na obtenção dos dados, que incluíram busca ativa das informações. Além disso, os resultados mostraram consistência 
com os obtidos por outros estudos que investigaram disparidades raciais na sobrevivência do câncer de mama e seus mediadores. Uma terceira limitação se relaciona com o uso da renda média domiciliar do setor censitário de residência da mulher como proxy da posição socioeconômica individual. Como a exposição de interesse foi raça/cor, e as variáveis relativas às condições socioeconômicas costumam ser muito correlacionadas entre si, provavelmente, a variável utilizada foi suficiente para o ajuste de confundimento, embora não se possa excluir a possibilidade de confundimento residual.

\section{Conclusão}

No Brasil, as condições de saúde das mulheres negras apresentam desigualdades estruturais, sendo a raça/cor um fator de exposição a pior acesso a bens, serviços e recursos sociais e de saúde. Neste estudo, o pior prognóstico das mulheres negras deveu-se, em grande parte, ao diagnóstico da doença em estadiamento mais avançado. Tal disparidade pode estar relacionada a desigualdades no acesso ao rastreamento mamográfico e ao diagnóstico precoce da doença. Isso aponta para a necessidade de ampliar a cobertura e a qualidade do programa de rastreamento e facilitar o acesso ao diagnóstico e tratamento precoces do câncer de mama, com vistas à redução da iniquidade racial.

\section{Colaboradores}

M. C. Nogueira, M. R. Guerra, e M. T. Bustamante-Teixeira contribuíram na concepção do estudo; análise e interpretação dos dados; redação do artigo e aprovação final da versão a ser publicada. J. R. D. Cintra, C. S. L. Corrêa, e V. A. Fayer contribuíram na concepção do estudo; análise e interpretação dos dados; revisão crítica relevante do conteúdo intelectual e aprovação final da versão a ser publicada.

\section{Agradecimentos}

Os autores agradecem às instituições de saúde envolvidas por toda a contribuição dispensada para a realização desta pesquisa.

\section{Referências}

1. Torre LA, Bray F, Siegel RL, Ferlay J, Lortettieulent J, Jemal A. Global cancer statistics, 2012. CA Cancer J Clin 2015; 65:87-108.

2. Ellis L, Canchola AJ, Spiegel D, Ladabaum U, Haile R, Gomez SL. Racial and ethnic disparities in cancer survival: the contribution of tumor, sociodemographic, institutional, and neighborhood characteristics. J Clin Oncol 2018; 36:25-33.

3. Jemal A, Robbins AS, Lin CC, Flanders WD, DeSantis CE, Ward EM, et al. Factors that contributed to black-white disparities in survival among nonelderly women with breast cancer between 2004 and 2013. J Clin Oncol 2018; 36:14-24

4. Newman LA, Griffith KA, Jatoi I, Simon MS, Crowe JP, Colditz GA. Meta-analysis of survival in African American and white American patients with breast cancer: ethnicity compared with socioeconomic status. J Clin Oncol 2006; 24:1342-9. 
5. Abreu ED, Koifman RJ, Fanqueiro AG, Gerardin M, Land P, Koifman S. Sobrevida de dez anos de câncer de mama feminino em coorte populacional em Goiânia (GO), Brasil, 19881990. Cad Saúde Colet (Rio J.) 2012; 20:30513.

6. Fayer VA, Guerra MR, Cintra JRD, Bustamante-Teixeira MT. Sobrevida de dez anos e fatores prognósticos para o câncer de mama na região Sudeste do Brasil. Rev Bras Epidemiol 2016; 19:766-78.

7. Guerra MR, Silva GA, Nogueira MC, Leite ICG, Oliveira RVC, Cintra JRD, et al. Sobrevida por câncer de mama e iniquidade em saúde. Cad Saúde Pública 2015; 31:1673-84.

8. Höfelmann DA, Anjos JC, Ayala AL. Sobrevida em dez anos e fatores prognósticos em mulheres com câncer de mama em Joinville, Santa Catarina, Brasil. Ciênc Saúde Coletiva 2014; 19:1813-24.

9. Schneider IJC, d'Orsi E. Sobrevida de cinco anos e fatores prognósticos em mulheres com câncer de mama em Santa Catarina, Brasil. Cad Saúde Pública 2009; 25:1285-96.

10. Laguardia J. Raça e epidemiologia: as estratégias para construção de diferenças biológicas. Ciênc Saúde Coletiva 2007; 12:253-61.

11. Moubarac JC. Persisting problems related to race and ethnicity in public health and epidemiology research. Rev Saúde Pública 2013; 47:105-16.

12. Chor D. Desigualdades em saúde no Brasil: é preciso ter raça. Cad Saúde Pública 2013; 29:1272-5.

13. Travassos C, Williams DR. The concept and measurement of race and their relationship to public health: a review focused on Brazil and the United States. Cad Saúde Pública 2004; 20:660-78.

14. Victora CG, Huttly SR, Fuchs SC, Olinto MT. The role of conceptual frameworks in epidemiological analysis: a hierarchical approach. Int J Epidemiol 1997; 26:224-7.

15. Hernán MA, Robins JM. Causal inference. Boca Raton: Chapman \& Hall/CRC; 2018.

16. Warner ET, Tamimi RM, Hughes ME, Ottesen RA, Wong YN, Edge SB, et al. Racial and ethnic differences in breast cancer survival: Mediating effect of tumor characteristics and sociodemographic and treatment factors. J Clin Oncol 2015; 33:2254-61.
17. Lange T, Vansteelandt S, Bekaert M. A simple unified approach for estimating natural direct and indirect effects. Am J Epidemiol 2012; 176:190-5.

18. Muniz JO, Bastos JL. Volatilidade classificatória e a (in)consistência da desigualdade racial. Cad Saúde Pública 2017; 33 Suppl $1: \mathrm{e} 00082816$.

19. Dignam JJ, Huang L, Ries L, Reichman M, Mariotto A, Feuer E. Estimating breast cancerspecific and other-cause mortality in clinical trial and population-based cancer registry cohorts. Cancer 2009; 115:5272-83.

20. Uno H, Claggett B, Tian L, Inoue E, Gallo P, Miyata T, et al. Moving beyond the hazard ratio in quantifying the between-group difference in survival analysis. J Clin Oncol 2014; 32:2380-5.

21. Carvalho M, Andreozzi V, Codeço C, Campos D, Barbosa M. Análise de sobrevivência: teoria e aplicações em saúde. 2a Ed. Rio de Janeiro: Editora Fiocruz; 2011.

22. Lange T, Hansen KW, Sørensen R, Galatius S. Applied mediation analyses: a review and tutorial. Epidemiol Health 2017; 39:e2017035.

23. Rochon J, du Bois A, Lange T. Mediation analysis of the relationship between institutional research activity and patient survival. BMC Med Res Methodol 2014; 14:9.

24. Freitas Júnior R, Nunes RD, Martins E, Curado MP, Freitas NMA, Soares LR, et al. Fatores prognósticos do câncer de mama e sobrevida global em cinco e dez anos na cidade de Goiânia, Brasil: estudo de base populacional. Rev Col Bras Cir 2017; 44:435-43.

25. Ferraz RO, Moreira-Filho DC. Análise de sobrevivência de mulheres com câncer de mama: modelos de riscos competitivos. Ciênc Saúde Coletiva 2017; 22:3743-54

26. Moraes ABD, Zanini RR, Turchiello MS, Riboldi J, Medeiros LRD. Estudo da sobrevida de pacientes com câncer de mama atendidas no hospital da Universidade Federal de Santa Maria, Rio Grande do Sul, Brasil. Cad Saúde Pública 2006; 22:2219-28.

27. Quinn MJ, Cooper N, Rachet B, Mitry E, Coleman MP. Survival from cancer of the breast in women in England and Wales up to 2001. Br J Cancer 2008; 99 Suppl 1:S53-5. 
28. Blamey RW, Hornmark-Stenstam B, Ball G, Blichert-Toft M, Cataliotti L, Fourquet A, et al. ONCOPOOL - a European database for 16,944 cases of breast cancer. Eur J Cancer 2010; 46:56-71.

29. Ahn SG, Lee HM, Lee SA, Jeong J, Lee H. Longterm survival analysis of Korean breast cancer patients at a single center: improving outcome over time. Yonsei Med J 2014; 55:1187-95.

30. Allemani C, Minicozzi P, Berrino F, Bastiaannet E, Gavin A, Galceran J, et al. Predictions of survival up to 10 years after diagnosis for European women with breast cancer in 20002002. Int J Cancer 2013; 132:2404-12.

31. Chor D, Lima CRA. Aspectos epidemiológicos das desigualdades raciais em saúde no Brasil. Cad Saúde Pública 2005; 21:1586-94.

32. VanderWeele TJ, Robinson WR. On the causal interpretation of race in regressions adjusting for confounding and mediating variables. Epidemiology 2014; 25:473-84.

33. Glass TA, Goodman SN, Hernán MA, Samet JM. Causal inference in public health. Ann Rev Public Health 2013; 34:61-75.

34. Mendonça GAS, Silva AM, Caula WM. Características tumorais e sobrevida de cinco anos em pacientes com câncer de mama admitidas no Instituto Nacional de Câncer, Rio de Janeiro, Brasil. Cad Saúde Pública 2004; 20:1232-9.

35. Li R, Daniel R, Rachet B. How much do tumor stage and treatment explain socioeconomic inequalities in breast cancer survival? Applying causal mediation analysis to population-based data. Eur J Epidemiol 2016; 31:603-11.

36. McKenzie F, Ives A, Jeffreys M. Socio-economic inequalities in survival from screendetected breast cancer in South West England: population-based cohort study. Eur J Public Health 2012; 22:418-22.

37. Rutherford MJ, Hinchliffe SR, Abel GA, Lyratzopoulos G, Lambert PC, Greenberg DC. How much of the deprivation gap in cancer survival can be explained by variation in stage at diagnosis: an example from breast cancer in the East of England. Int J Cancer 2013; 133:2192200.
38. Woods LM, Rachet B, Coleman MP. Origins of socio-economic inequalities in cancer survival: a review. Ann Oncol 2006; 17:5-19.

39. Smith EC, Ziogas A, Anton-Culver H. Delay in surgical treatment and survival after breast cancer diagnosis in young women by race/ethnicity. JAMA Surg 2013; 148:516-23.

40. Nurgalieva ZZ, Franzini L, Morgan RO, Vernon SW, Liu CC, Du XL. Impact of timing of adjuvant chemotherapy initiation and completion after surgery on racial disparities in survival among women with breast cancer. Med Oncol 2013; 30:419.

41. Yang R, Cheung MC, Franceschi D, Hurley J, Huang Y, Livingstone AS, et al. African-American and low-socioeconomic status patients have a worse prognosis for invasive ductal and lobular breast carcinoma: do screening criteria need to change? J Am Coll Surg 2009; 208:85368.

42. Leal MC, Gama SGN, Frias P, Szwarcwald CL. Healthy lifestyles and access to periodic health exams among Brazilian women. Cad Saúde Pública 2005; 21 Suppl 1:S78-88.

43. Kumar P, Aggarwal R. An overview of triplenegative breast cancer. Arch Gynecol Obstet 2016; 293:247-69.

44. Sail K, Franzini L, Lairson D, Du X. Differences in treatment and survival among African-American and Caucasian women with early stage operable breast cancer. Ethn Health 2012; 17:309-23.

45. Barata RB, Almeida MF, Montero CV, Silva ZP. Health inequalities based on ethnicity in individuals aged 15 to 64, Brazil, 1998. Cad Saúde Pública 2007; 23:305-13.

46. Laguardia J. O uso da variável "raça" na pesquisa em saúde. Physis (Rio J.) 2004; 14:197234. 


\section{Abstract}

The study's objectives were to investigate the association between race/color and 10-year survival in women with breast cancer and the role of staging as mediator. This was a hospital cohort with 481 women with invasive breast cancer diagnosed from 2003 to 2005. Comparisons were made between white and black women as to sociodemographic characteristics and staging, using the chisquare test, and 10-year survival, using KaplanMeier and Cox regression methods. For the race/ color variable, direct and indirect effects were estimated, mediated by staging, with adjustment for the socioeconomic status of the woman's area of residence and age, using the potential responses (counterfactual) model and Cox multiple regression. Black women living in low-income census tracts were more likely to use the public health care system and to be diagnosed at more advanced stages. Breast cancer-specific 10-year survival was 64.3\% (95\%CI: 60.0; 68.9), with a significant difference between whites (69.5\%; 95\%CI: 64.8; 74.6) and blacks (44; 95\%CI: 35.2\%; 55.1). In the multivariate models adjusted for income and age, black women had worse prognosis $(\mathrm{HR}=2.09 ; 95 \% \mathrm{CI}$ : $1.76 ; 2.51)$, and the proportion mediated by staging was 40\% (95\%CI: 37; 42). There is racial disparity in 10-year breast cancer survival in Brazilian women, mediated mainly by more advanced staging at diagnosis in black women. This highlights the need to expand both the coverage and the quality of breast cancer screening and to facilitate access to early diagnosis and treatment in order to reduce racial inequality.

Health Equity; Ethnicity and Health; Breast Neoplasms; Survival Analysis

\section{Resumen}

Los objetivos fueron investigar la asociación entre raza/color y la supervivencia en 10 años de mujeres con cáncer de mama y el papel de la fase de desarrollo como mediador. Se trata de una cohorte hospitalaria de 481 mujeres, con cáncer invasivo de mama, diagnosticadas entre 2003 y 2005. Se realizaron comparaciones entre mujeres blancas y negras, en cuanto a las características sociodemográficas y a la fase del cáncer, usando el test chi-quadrado, y la supervivencia en 10 años, usando los métodos de Kaplan-Meier y regresión de Cox. Se estimaron para la variable raza/color efectos directos e indirectos, mediados por las diferentes fases, con ajuste en la condición social del área de residencia y edad, utilizando el modelo de respuestas potenciales (contrafactual) y regresión múltiple de Cox. Las mujeres negras residian en sectores censitarios de menor renta, eran usuarias del sector público en mayor proporción y fueron diagnosticadas en fases más avanzadas. La supervivencia específica en 10 años fue de un 64,3\% (IC95\%: 60,0; 68,9), con una diferencia significativa entre blancas (69,5\%; IC95\%: 64,8\%-74,6\%) y negras (44\%; IC95\%: 35,2; 55,1). En los modelos múltiples, ajustados para renta y edad, las negras tuvieron un peor pronóstico (HR = 2,09; IC95\%: 1,76-2,51), y la proporción mediada por el estadio fue de un 40\% (IC95\%: 37; 42). Existe disparidad racial en la supervivencia del cáncer de mama en 10 años, mediada principalmente por el estadio más avanzado de la enfermedad en las mujeres negras. Esto apunta la necesidad de ampliar la cobertura y calidad del programa de rastreo de esa enfermedad y facilitar el acceso al diagnóstico y tratamiento precoces, con vistas a la reducción de la inequidad racial.

Equidad en Salud; Origen Étnico y Salud;

Neoplasias de la Mama; Análisis de

Supervivencia
Recebido em 06/Dez/2017

Versão final reapresentada em 20/Abr/2018

Aprovado em 02/Mai/2018 